\title{
Cerebral Amyloid Angiopathy with Lobar Haemorrhages and CAA-Related Inflammation in an Indian Family
}

\author{
Boby Varkey Maramattom \\ Division of Neurocritical Care, Department of Neurology, Aster Medcity, Kothad, Kochi, India
}

\section{Keywords}

Hereditary cerebral amyloid angiopathy · Cerebral amyloid angiopathy · Familial cerebral amyloid angiopathy .

Hereditary cerebral amyloid angiopathy in India

\begin{abstract}
Introduction: Cerebral amyloid angiopathy (CAA) is a common cause of lobar intracerebral haemorrhage. Sporadic CAA is far more common than hereditary CAA ( $h-C A A)$. Familial CAA has not yet been described from India. Case Report: Two elderly Indian women (a mother and daughter) presented 7 years apart with features of CAA. The mother had presented with features of CAA-related inflammation that responded to steroids, whereas the daughter presented with features of CAA-related intracerebral haemorrhage. Clinical exome testing did not reveal any known genetic variants associated with h-CAA. Discussion: Although clinical exome testing was inconclusive, the presentation of CAA in two generations (mother and daughter) in their 8th and 7th decades, respectively, raises the possibility of a familial CAA rather than a sporadic CAA in this Indian family. Genome-wide association studies are necessary to reveal if an Indian variant of familial CAA exists. We compare and contrast our familial CAA with the described h-CAA variants in the literature.

(c) 2022 The Author(s)

Published by S. Karger AG, Basel
\end{abstract}

\section{Introduction}

Cerebral amyloid angiopathy (CAA) is a common cause of lobar intracerebral haemorrhage (ICH). The pathological deposition of amyloid fibrils in the media and adventitia of small- to medium-sized cortical and leptomeningeal arteries causes a small-vessel vasculopathy. Fusiform enlargement and disruption of the lobar arterioles by amyloid causes lobar ICH [1]. Other MRI findings include cortical micro-haemorrhages, focal convexity subarachnoid haemorrhage, and cortical superficial siderosis. Amyloid deposits also lead to progressive arteriolar luminal obliteration and microvascular occlusion producing ischaemic CAA lesions, including small cortical infarcts, diffuse white matter ischaemia, and leukoencephalopathy. Infrequently, vascular inflammation directed against the amyloid in CAA results in acute or subacute encephalopathy. This CAA-associated inflammation/angiitis-related encephalopathy presents with subacute cognitive decline, seizures, and headache and maybe is steroid-responsive.

CAA-associated inflammation is of 2 types: amyloid beta protein $(A \beta)$-related angiitis (angio-destructive inflammation) or CAA-related inflammation (CAA-RI or perivascular inflammation) and are distinguishable by biopsy findings. Criteria for the diagnosis of CAA-RI in-
(C) 2022 The Author(s).

Published by S. Karger AG, Basel

This is an Open Access article licensed under the Creative Commons Attribution-NonCommercial-4.0 International License (CC BY-NC) (http://www.karger.com/Services/OpenAccessLicense), applicable to the online version of the article only. Usage and distribution for commercial purposes requires written permission.
Correspondence to:

Boby Varkey Maramattom, bobvarkey@gmail.com 






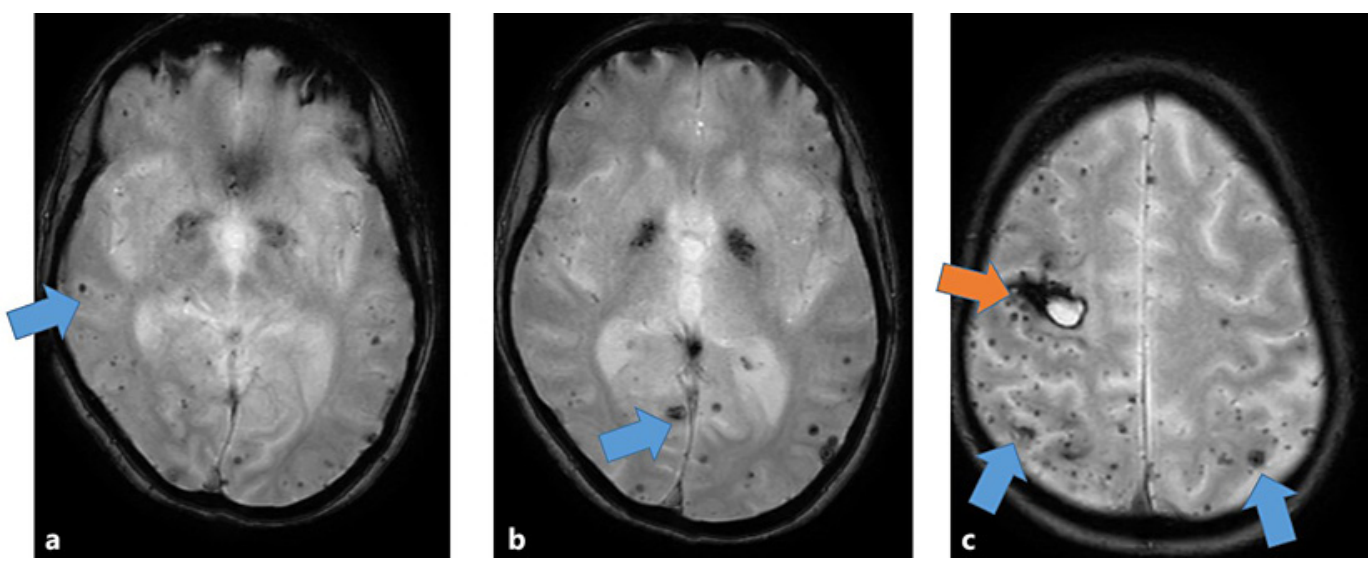

Fig. 1. GRE axial MRI images of the daughter (case 1). a, b Multiple scattered cortical microhaemorrhages (blue arrows). c Hemosiderin-lined old ICH (orange arrow).

clude the acute or subacute onset of symptoms; age $\geq 40$ years; one or more of the following symptoms: headache, mental or behavioural change, focal neurological signs, or seizures; MRI showing patchy or confluent T2 signal; evidence of CAA by gradient-echo MRI sequences or previous lobar haemorrhage; and no neoplastic or infectious explanation for these findings.

Sporadic CAA is far more common than hereditary CAA (h-CAA). h-CAA presents with dementia, lobar haemorrhage, or leukoencephalopathy and often starts in mid-adulthood. The different variants of h-CAA are named according to the regions where they are first diagnosed and are sub-characterized by their genetic abnormalities. The common h-CAAs are the Dutch, Flemish, Italian, and Icelandic CAA as well as the familial British dementia and familial Danish dementia (Table 1). The study described two women (mother and daughter) who developed late-onset CAA (7th and 8th decades). Although clinical exome testing was inconclusive, the presentation in two successive generations was suggestive of a familial CAA.

\section{Case Reports}

Case 1

A 67-year-old woman presented with an acute confusional state, 5 weeks after a left hemiparesis. CT of the brain had shown a right frontal lobar haemorrhage, and she improved spontaneously. She was started on carbamazepine for possible left sensory seizures and became excessively drowsy with incoherent speech. She was admitted, and carbamazepine was discontinued. MRI of the brain showed multiple lobar microhaemorrhages and a focus of hemosiderin-lined gliotic area at the location of the old $\mathrm{ICH}$ (Fig. 1). A diagnosis of CAA with "amyloid spells" was made. She recovered in 5 days. Neuropsychological evaluation after 3 weeks showed mild cognitive dysfunction (MMSE 24/30 and Addenbrooke cognitive exam score of $85 / 100$ ). Her mother had previously been examined by me 7 years earlier. Her clinical details were also retrieved. There was no other family history of ICH or dementia.

\section{Case 2}

Her mother had been admitted 7 years earlier at the age of 77 years with an encephalopathy, right hemiparesis, and aphasia that had evolved over a week. On examination, she was conscious, aphasic, and had grade 0/5 MRC power on the right side. MRI FLAIR, GRE, and T1 post-contrast sequences had shown multiple non-enhancing bilateral cerebral white matter lesions with central micro-haemorrhages (Fig. 2). A provisional diagnosis of CAA or multiple metastases had been made at that time. $\mathrm{CT}$ of the thorax and abdomen were normal, and she was treated with IV methylprednisolone $1 \mathrm{~g} /$ day for 5 days. They had refused a brain biopsy at that time. She made a gradual recovery and became ambulant over 2 months. She had no further neurological events and was still living alone and independently without any cognitive impairment at the age of 84 years (MMSE $27 / 30$ and $A C E 86 / 100$ ) at the time of this report. A review of her MRI images retrospectively, suggested the diagnosis of CAA-RI due to the extensive areas of vasogenic oedema involving the subcortical white matter, large areas of lobar white matter oedema, foci of cortical/subcortical microbleeds, overlying leptomeningeal enhancement, and subcortical contrast enhancement [2].

Clinical exome testing in both cases suggested variants of unknown significance (VUS) for APOA4 and $\mathrm{NOTCH} 3$ genes (which are found in CADASIL). However, after discussion with a geneticist and comparison with diseases databases (dbSNP, ClinVar, OMIM, and HGMD), these VUS were deemed insignificant. 

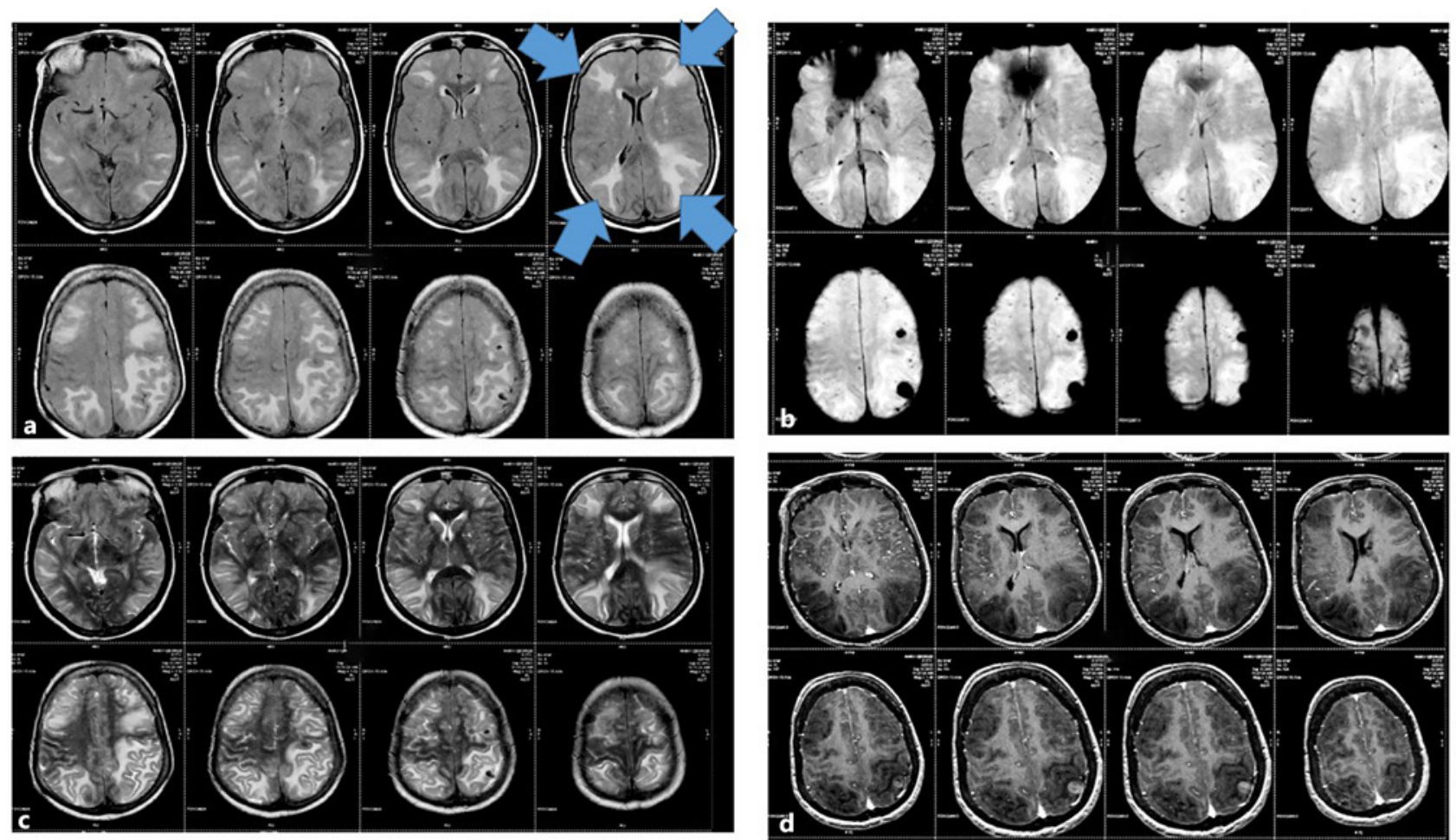

Fig. 2. Axial MRI sequences: FLAIR (a), GRE (b), T2 WI (c), and post-contrast T1-weighted images (d), showing bilateral extensive subcortical white matter oedema with cortical microhaemorrhages (b) and left parietal contrast enhancement $(\mathbf{d})$.

\section{Discussion}

This case report presented two South Indian women (mother and daughter) who presented with features of CAA and CAA-RI in their 7th and 8th decades respectively. The patient's mother fulfilled the clinical-radiological diagnostic criteria for CAA-RI [3]. The apolipoprotein A genotype, especially ApoA1, is rarely associated with CAA [4]. However, APOA2, APOE, or APOA4 have not yet been associated with CAA, although their protein products are amyloidogenic and detectable within amyloid deposits. Similarly, cerebral autosomal dominant arteriopathy with subcortical infarcts and leukoencephalopathy (CADASIL) and CAA are distinct cerebrovascular angiopathies with different clinical presentations. Nevertheless, they exhibit a considerable overlap in protein accumulation profiles and potentially a common mechanism of small vessel vasculopathy [5]. Patients with CADASIL are more likely to have cerebral microbleeds (31-69\%), and up to $25 \%$ of symptomatic patients with
CADASIL have intracerebral haemorrhages. However, large lobar haemorrhages are rare in CADASIL [6].

CAA-RI is also not a feature of CADASIL as it is due to a different pathological process than CAA (deposition of non-amyloidogenic granular osmiophilic materials in the media of small arteries and arterioles rather than amyloid deposits).

$\mathrm{h}$-CAA is rare compared to sporadic CAA and is characterized by an earlier age of onset and a more severe course of illness. h-CAA are often autosomal dominant in inheritance and manifest with ischaemic or haemorrhagic cerebrovascular manifestations. Broadly, they can be divided into $A \beta$ ( $A \beta-C A A)$ and non- $A \beta$ (non- $A \beta$ CAA) forms. The A $\beta$ forms which are derived from defects in the processing of the $\beta$-amyloid precursor protein often present with lobar haemorrhage. Non-A $\beta$ forms usually present with dementia or leukoencephalopathy and rarely develop ICH [7]. The Icelandic non-A $\beta$ is an exception with its predilection to produce lobar ICH in very young individuals.
Cerebrovasc Dis Extra 2022;12:23-27 DOI: $10.1159 / 000522214$
Maramattom 


\section{Conclusions}

Two generations of an Indian family presented with a haemorrhagic CAA. Although genetic testing was inconclusive, the presentation of CAA in two successive generations, suggested a familial aetiology. The lack of dementia and the presence of CAA-RI (in the mother) differentiated our cases from the Caucasian variants of h-CAA.

Although CAA-RI has not been described in h-CAA, the occurrence of CAA-ICH in two generations makes familial CAA more likely than sporadic CAA or CADASIL. In view of the lobar haemorrhage in our patients, we speculate that they might harbour an A $\beta$-CAA. However, only genome-wide association studies may be able to identify the genes responsible for our type of familial CAA in India. Our study was limited by the inability to obtain brain biopsy samples for further analysis. However, recognition of the existence of an Indian variant of familial CAA will spur genetic studies to characterize the genes responsible.

\section{Statement of Ethics}

EC approval was not necessary from our institution as it involved less than 3 patients. Ethical approval for use of these samples for research purposes was not required for this study in ac- cordance with hospital guidelines. Images presented in this study were obtained as part of routine medical care. Written informed consent was obtained from the patients for the publication of the case reports and the accompanying images.

\section{Conflict of Interest Statement}

The author has no conflict of interest regarding the publication of the manuscript.

\section{Funding Sources}

No funding was taken.

\section{Author Contributions}

The author is responsible for concept, draft, writing, and editing.

\section{Data Availability Statement}

All data generated or analysed during this study are included in this article. Further enquiries can be directed to the corresponding author.

\section{References}

1 Yamada M. Cerebral amyloid angiopathy: emerging concepts. J Stroke. 2015;17:17-30.

2 Corovic A, Kelly S, Markus HS. Cerebral amyloid angiopathy associated with inflammation: a systematic review of clinical and imaging features and outcome. Int J Stroke. 2018; 13:257-67.

3 Chung KK, Anderson NE, Hutchinson D, Synek B, Barber PA. Cerebral amyloid angiopathy related inflammation: three case reports and a review. J Neurol Neurosurg Psychiatry. 2011;82:20-6.

4 Camacho J, Moliné T, Bonaterra-Pastra A, Ramón Y Cajal S, Martínez-Sáez E, Hernández-Guillamon M. Brain ApoA-I, ApoJ and ApoE immunodetection in cerebral amyloid angiopathy. Front Neurol. 2019;13;187.
5 Young KZ, Xu G, Keep SG, Borjigin J, Wang MM. Small blood vessel disease in the brain theme issue overlapping protein accumulation profiles of CADASIL and CAA: is there a common mechanism driving cerebral small vessel disease? Am J Pathol. 2020;30:S0002944030597-6.

6 Choi JC, Kang SY, Kang JH, Park JK. Intracerebral hemorrhages in CADASIL. Neurology. 2006;67:2042-4.

7 Biffi A, Greenberg SM. Cerebral amyloid angiopathy: a systematic review. J Clin Neurol. 2011;7:1-9.

8 Maat-Schieman M, Roos R, van Duinen S. Hereditary cerebral hemorrhage with amyloidosis-Dutch type. Neuropathology. 2005;25: 288-97.
9 Sellal F, Wallon D, Martinez-Almoyna L, Marelli C, Dhar A, Oesterlé H, et al. APP mutations in cerebral amyloid angiopathy with or without cortical calcifications: report of three families and a literature review. J Alzheimers Dis. 2017;56:37-46.

10 Grabowski TJ, Cho HS, Vonsattel JP, Rebeck GW, Greenberg SM. Novel amyloid precursor protein mutation in an Iowa family with dementia and severe cerebral amyloid angiopathy. Ann Neurol. 2001;49:697-705.

11 Palsdottir A, Snorradottir AO, Thorsteinsson L. Hereditary cystatin $C$ amyloid angiopathy: genetic, clinical, and pathological aspects. Brain Pathol. 2006;16(1):55-9. 